\title{
Liver Fluke-Associated Biliary Tract Cancer
}

Piyapan Prueksapanich, Panida Piyachaturawat, Prapimphan Aumpansub, Wiriyaporn Ridtitid, Roongruedee Chaiteerakij, and Rungsun Rerknimitr

Division of Gastroenterology, Department of Medicine, Chulalongkorn University Faculty of Medicine, Bangkok, Thailand

Cholangiocarcinoma (CCA) is an aggressive cancer arising from epithelial cells of the bile duct. Most patients with CCA have an unresectable tumor at the time of diagnosis. In Western countries, the risk of CCA increases in patients with primary sclerosing cholangitis, whereas liver fluke infection appears to be the major risk factor for CCA in Asian countries. A diagnosis of liver fluke infection often relies on stool samples, including microscopic examination, polymerase chain reaction-based assays, and fluke antigen detection. Tests of serum, saliva and urine samples are also potentially diagnostic. The presence of liver fluke along with exogenous carcinogens magnifies the risk of CCA in people living in endemic areas. The "liver fluke-cholangiocarcinoma" carcinogenesis pathways consist of mechanical damage to the bile duct epithelium, immunopathologic and cellular reactions to the liver fluke's antigens and excretory/secretory products, liver fluke-induced changes in the biliary tract microbiome and the effects of repeated treatment for liver fluke. A vaccine and novel biomarkers are needed for the primary and secondary prevention of CCA in endemic areas. Importantly, climate change exerts an effect on vector-borne parasitic diseases, and awareness of liver fluke should be enhanced in potentially migrated habitat areas. (Gut Liver 2018;12:236245)

Key Words: Cholangiocarcinoma; Opisthorchiasis; Clonorchiasis

\section{INTRODUCTION}

Cholangiocarcinoma (CCA) is a catastrophic malignant neoplasm of the bile duct. In Western countries, the risk of CCA increases in patients with primary sclerosing cholangitis, whereas liver fluke infection appears to be the major risk factor for CCA in Asian countries.

\section{BILE DUCT CANCER (CHOLANGIOCARCINOMA)}

\section{Clinical presentations}

CCA, the second most common primary liver cancers, is an aggressive cancer arising from malignant transformation of biliary epithelial cells. ${ }^{1}$ Globally, CCA accounts for approximately $10 \%$ to $20 \%$ of primary liver cancers. ${ }^{2}$ Based on anatomical structures, CCA is divided into two subtypes: extrahepatic and intrahepatic, which accounts for approximately 75\% and 25\% of all cases, respectively. ${ }^{3}$ Extrahepatic CCA can be further classified into perihilar, middle, and distal, depending on the locations of the tumor. ${ }^{4,5}$ The perihilar CCA is further categorized according to the Bismuth-Corlette classification as addressed in the literature. ${ }^{6}$ The clinical presentations of CCA range from nonspecific symptoms to signs related to late stages of disease. Anatomical subtypes of CCA may affect clinical presentations of patients. Extrahepatic CCA often presents with signs of bile duct obstruction such as painless jaundice, pruritus, pale stools and dark urine. Intrahepatic CCA commonly presents with nonspecific symptoms such as fatigue, abdominal pain, liver mass, and weight loss. Nevertheless, some asymptomatic patients with CCA are incidentally diagnosed by imaging studies during a routine health check-up. Most CCA cases presented as unresectable tumor with stage III or stage IV at the time of diagnosis. ${ }^{3}$ Therefore, CCA is unfortunately associated with poor prognosis and unfavorable treatment outcomes.

\section{Risk factors}

Due to differences in risk factors and genetics in various regions, the incidence rates of CCA vary geographically. The highest incidence rates are documented in sub-Saharan Africa, Eastern and South-Eastern Asia. ${ }^{7}$ In Eastern and South-

Correspondence to: Rungsun Rerknimitr

Division of Gastroenterology, Department of Medicine, Chulalongkorn University Faculty of Medicine, 1873 Rama 4 Road, Pathumwan district, Bangkok 10330, Thailand

Tel: +66-2-256-4265, Fax: +66-2-256-4265, E-mail: ERCP@live.com

Received on March 2, 2017. Revised on April 29, 2017. Accepted on May 6, 2017. Published online August 9, 2017 pISSN 1976-2283 eISSN 2005-1212 https://doi.org/10.5009/gnl17102

(c) This is an Open Access article distributed under the terms of the Creative Commons Attribution Non-Commercial License (http://creativecommons.org/licenses/by-nc/4.0) which permits unrestricted non-commercial use, distribution, and reproduction in any medium, provided the original work is properly cited. 
Table 1. Risk Factors in Patients Presenting with Cholangiocarcinoma ${ }^{9}$

\begin{tabular}{ll}
\hline Established risk factor & \multicolumn{1}{c}{ Possible risk factor } \\
\hline Liver flukes infection & Hepatitis B, C and cirrhosis \\
Opisthorchis viverrini & \\
Clonorchis sinensis & \\
Schistosomiasis japonica & \\
Primary sclerosing cholangitis & Diabetes \\
Hepatolithiasis & Obesity \\
Toxic agents & Alcohol \\
Bile duct cyst & Smoking \\
Caroli's disease & \\
Choledochal cyst & \\
\hline
\end{tabular}

Eastern Asia, the incidence of CCA is relatively low in Korea and Japan whereas the incidence rate of CCA in Thailand is extremely high with age-standardized incidence rates of 33.4 per 100,000 in men and 12.3 per 100,000 in women. ${ }^{7}$ Known risk factors are established among a minority of patients with CCA (Table 1). ${ }^{5}$ In Western countries, the risk of CCA increases in patients with primary sclerosing cholangitis whereas liver fluke infection (Opisthorchis viverrini, Clonorchis sinensis, and Schistosomiasis japonica) appears to be the major risk factor of CCA in Asian countries. ${ }^{5,7-9}$ Exposure to toxic compounds such as Thorotrast has also been related to an increased risk of CCA. ${ }^{9}$ Further, previous series showed several diseases associated with the development of CCA including hepatitis B and C infection, cirrhosis, obesity and diabetes mellitus. ${ }^{9}$ In Thailand, the highest percentage of CCA was observed in the north-eastern part where the highest prevalence of $O$. viverrini was reported in this country. ${ }^{7,10}$ Based on the World Health Organization (WHO), $O$. viverrini has also been addressed as class I carcinogen. ${ }^{11}$

\section{LIVER FLUKE}

\section{The life cycle}

$O$. viverrini and the related liver fluke $C$. sinensis, food-borne trematodes, have similar life cycles which starts when humans and other fish-eating mammals, ${ }^{12}$ the definitive hosts, passed eggs through their feces into the environment. When eggs reach freshwater, they hatch and the miracidia infect their first intermediate host, a freshwater snail (Bithynia spp. or Parafossarulus spp.), ${ }^{13}$ where they transform into sporocysts, rediae, and cercariae. Cercariae escape from the snail and penetrate freshwater fish (Cyclocheilichthys spp., Puntius spp., Hampala dispar), ${ }^{14}$ which are the second intermediate host. The cercariae encyst as metacercariae in the muscles or under the scales which can infect humans by eating raw or undercooked cyprinoid fish products. ${ }^{15}$ The metacercariae reach human small intestine unharmed and migrate through the ampulla of Vater, they reach and inhabit in the bile ducts, where they eventually mature into adult worms within 4 weeks and deposit yellow, operculated eggs. The parasites may live for up to 25 years in human body. ${ }^{16}$

\section{Geographical distribution}

The global estimate of the number of people infected with liver fluke is 45 million: 35 million with $C$. sinensis (15 million in China) and 10 million with 0 . viverrini (8 million in Thailand). ${ }^{17-20}$ As many as 700 million people worldwide are at risk of infection by the liver flukes. Both infections are endemic in the Far East, Southeast Asia, and Eastern Europe. C. sinensis is endemic in northeast China, southern Korea, Japan, Taiwan, northern Vietnam, and the far eastern part of Russia, whereas $O$. viverrini is endemic in Southeast Asia countries, including Laos, Cambodia, Thailand, Vietnam, and with some reported cases in Malaysia, Singapore and the Philippines.

\section{DIAGNOSTIC TEST FOR LIVER FLUKE INFECTION}

\section{Stool specimen}

The microscopic method of flukes' eggs detection in stool is typically used as a diagnostic test because of its availability and inexpensiveness. However, burden of disease does affect the accuracy of the test. The lower burden of fluke, the lower sensitivity of stool parasitological test. ${ }^{21}$ In addition, liver fluke and other intestinal flukes infections can be superimposed and their eggs (Opisthorchis-like eggs) could be difficult to be microscopically differentiated. $^{22-24}$

The polymerase chain reaction (PCR)-based method for detection of liver fluke's genetic materials in stool could diagnose opisthorchiasis in $28.6 \%$ to $76.6 \%$ of cases in which stool microscopic examinations were negative. ${ }^{25-27}$ Moreover, PCR-based method could be used to differentiate 0 . viverrini from other Opisthorchis-like eggs. ${ }^{28-30}$ As compared to purge results, PCR test of $O$. viverrini had sensitivities of 93.7\%. ${ }^{31}$ However, the PCR-based test could be falsely negative due to the presence of PCR inhibitor in stool. ${ }^{25-27}$ A deoxyribonucleic acid (DNA) extraction protocol using cetyltrimethylammonium bromide to remove inhibitors had been used to optimize the sensitivity. ${ }^{25}$ The sensitivity of PCR-based stool test, which was found to be as low as one-half in the specimens containing less than 200 eggs per gram of feces, also depended on the intensity of $O$. viverrini infection. $^{32}$

In addition to liver fluke's genetic material, the 0 . viverrini antigens could also be discovered in stool (coproantigen). The detection of $O$. viverrini cathepsin $\mathrm{F}$ by a sandwich enzymelinked immunosorbent assay (ELISA) assay yielded a sensitivity and a specificity of 93.3\% and 78.5\%, respectively, in a hamster model study. ${ }^{33}$ A monoclonal antibody-based ELISA (Mab-ELISA) could also be used to detect $O$. viverrini metabolic antigen in feces. ${ }^{34}$ The sensitivity and specificity of trichloroacetic acid (TCA)-based Mab-ELISA were 97.9\% and 54.2\%, respectively. The TCA-based Mab-ELISA were positive in about a half of 
specimens which were negative for egg detection. ${ }^{35}$

\section{Other specimens (serum, urine, saliva)}

The circulating liver fluke's antigens could be found in human serum and could be used as a diagnostic tool for liver fluke infection. ${ }^{36}$ O. viverrini excretory/secretory products (OvESP) were also detected in urine. The sensitivity and specificity of the urine OvESP assay were 81\% and 70\%, respectively, when compared to the stool examination with a formalin-ether concentration technique. ${ }^{37}$

Detection of liver fluke's specific antibodies in serum, saliva and urine could be performed. ${ }^{33,38,39}$ However, a presence of the antibody could not be used to distinguish between a past and a current infection.

\section{THE CURRENT TREATMENT OF LIVER FLUKE INFECTION}

Praziquantel is a drug of choice for treatment of fluke infection including opisthorchiasis and clonorchiasis. The standard dose of praziquantel is $75 \mathrm{mg} / \mathrm{kg}$ in three divided doses for 1 day, which results in an egg reduction rate of $98 \%$ to $99 \%$ for both 0 . viverrini and $C$. sinensis infection. ${ }^{40}$ The adverse events of praziquantel treatment, such as dizziness, headache, and nausea, are uncommon.

Several novel agents for treatment of liver fluke have been recently proposed. Tribendimidine is one of the promising agents. Tribendimidine is an amidantel derivative which possesses activities against not only liver fluke but also some intestinal roundworm such as hookworms, ascariasis and enterobiasis. ${ }^{41,42}$ Patients in endemic areas with co-infection of liver fluke and roundworms would benefit from the broad-spectrum coverage of tribendimidine. The egg reduction rate of a 400-mg single dose tribendimidine treatment against both $O$. viverrini $i^{41,43}$ and C. sinensis ${ }^{44}$ was $98 \%$ to $99 \%$, which was comparable to that of praziquantel but with less adverse effects. The most common adverse effects of tribendimidine were dizziness, vertigo, headache, nausea, and fatigue. ${ }^{43,44}$

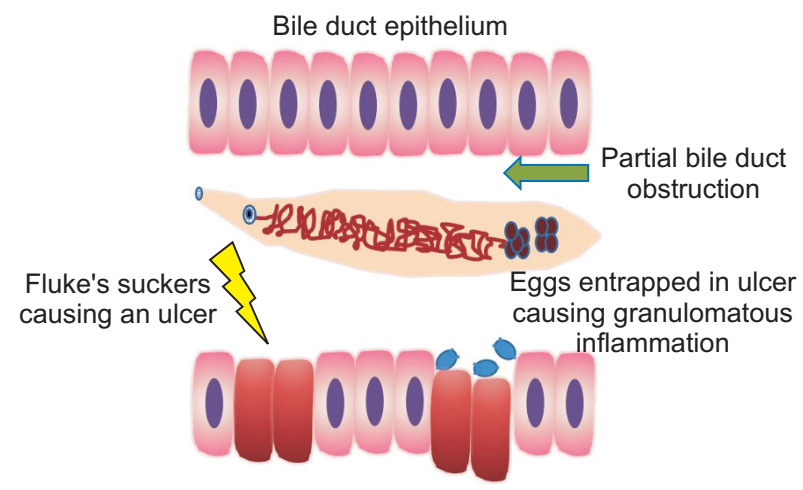

Fig. 1. Mechanical damage to the bile duct epithelium caused by liver fluke.

\section{LIVER FLUKE-ASSOCIATED CARCINOGENESIS OF BILE DUCT CANCER}

O. viverrini-related CCA possesses distinct signatures of genetics, epigenetics and transcriptional profiles comparing to non-O. viverrini-related CCA. ${ }^{45}$ Some major pathways that link liver fluke to the development of CCA have been proposed. ${ }^{46}$ However, many promising pathways are continuously emerging from novel "omics" technologies such as proteomics, ${ }^{47}$ genomics ${ }^{48}$ and miRNAomics. ${ }^{49,50}$ The combination of these mechanisms and exogenous carcinogens such as nitrosamines in fermented fish and pork magnify risk of CCA in people living in endemic areas.

\section{Mechanical damage}

Bile duct epitheliums could be physically injured by liver flukes' suckers that cling to the bile duct wall, particularly to the medium- and large-sized bile ducts, causing bile duct ulcers. In addition, the flukes' eggs could be ensnared into those ulcers inducing a granulomatous inflammation of the periductal tissue (Fig. 1). ${ }^{51}$ In case of $C$. sinensis, of which the size is larger than $O$. viverrini, the fluke causes a partial bile duct obstruction resulting in bile stasis and an increase in biliary pressure, due to the relatively large size of the fluke as compared to human bile duct. $^{52}$ These repeated circles of ulceration, inflammation and healing process eventually result in DNA damage and the development of CCA.

\section{Immunopathology}

On top of the physical damage from the flukes, human immune reaction to the parasites causing more damage to its biliary epithelium (Fig. 2). There are a number of immune mechanisms to $O$. viverrini infection including the fluke-specific $\mathrm{IgG}^{53}$ and non-fluke specific immune response. One of the most important inflammatory cytokines is interleukin-6 (IL-6). The high level of circulating plasma IL- 6 is associated with the degree of advanced periductal fibrosis from chronic 0 . viverrini infection but not with the infection with 0 . viverrini itself. ${ }^{54}$ In endemic areas of liver fluke infection, the higher plasma IL-6 level, the greater risk of developing advanced periductal fibrosis and CCA. ${ }^{54,55}$ IL- 6 can also promote CCA cell line proliferation by activation of human progranulin expression. ${ }^{56}$

The mechanisms by which $O$. viverrini induces host immune response have been demonstrated in normal immortalized human cholangiocyte cell line (H69) and human CCA cell line (KKU-100, KKU-M156) studies. The OvESP were endocytosed readily by normal cholangiocyte cells and induced proliferation of both normal cholangiocytes and CCA cells. ${ }^{57}$ The OvESP also induced inflammatory cascade by upregulation of Toll-like receptors (TLR) 4, activation of nuclear factor $-\kappa \mathrm{B}(\mathrm{NF}-\kappa \mathrm{B})$ and expression and secretion of both IL- 6 and IL-8. After activation of NF- $\kappa \mathrm{B}$, the inducible nitric oxide synthase (iNOS) and cyclo- 


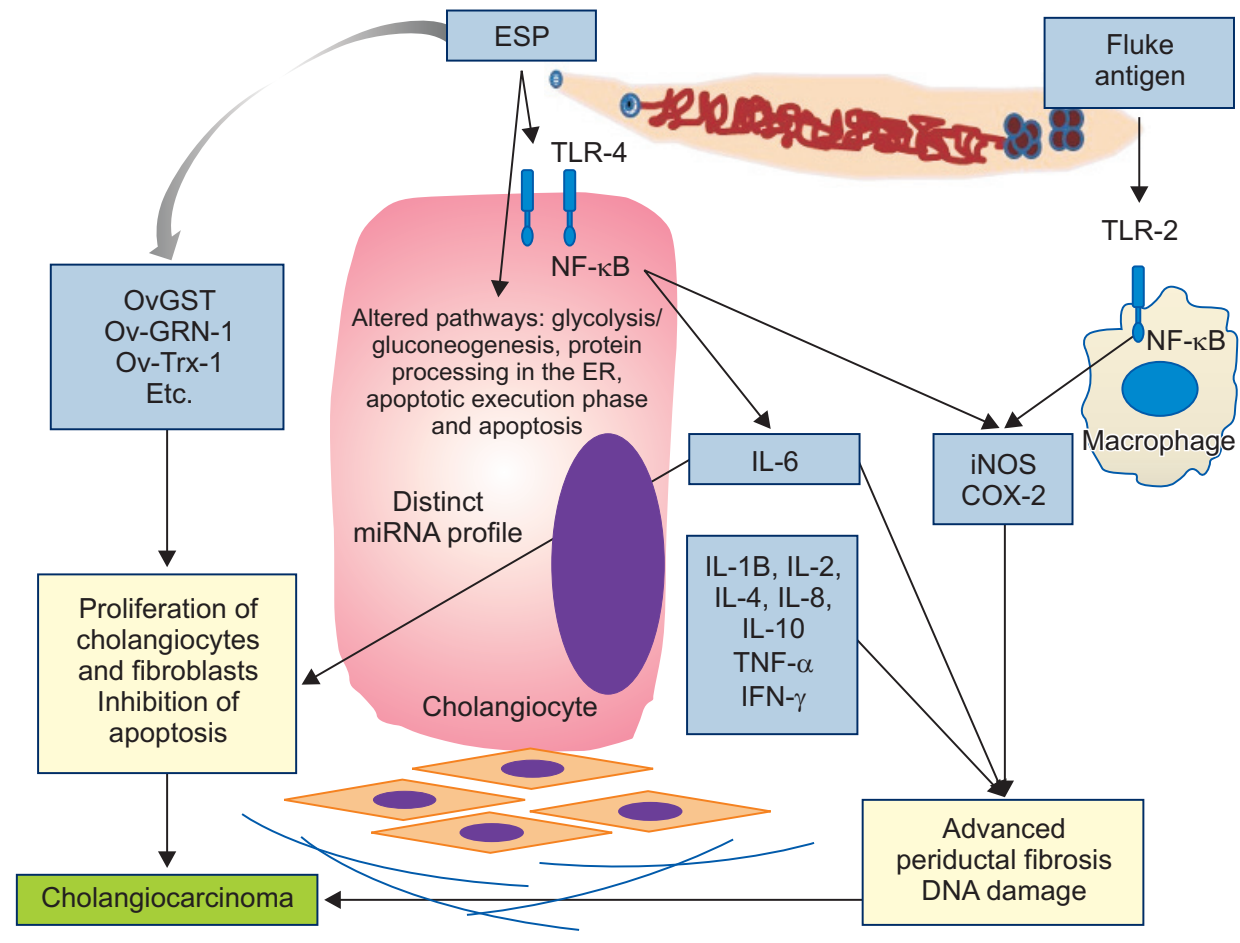

Fig. 2. Immunopathologic reactions of human cells to liver fluke infection.

ESP, excretory/secretory products; OvGST, Opisthorchis viverrini glutathione S-transferase; Ov-GRN-1, $O$. viverrini granulin; Ov-Trx-1, $O$. viverrini thioredoxin; TLR, Toll-like receptors; $\mathrm{NF}-\mathrm{\kappa} \mathrm{B}$, nuclear factor- $\mathrm{\kappa} \mathrm{B}$; IL, interleukin; TNF, tumor necrosis factor; IFN, interferon; iNOS, inducible nitric oxide synthase; COX-2, cyclooxygenase-2; miRNA, microribonucleic acid; ER, endoplasmic reticulum. oxygenase-2 (COX-2) are stimulated. ${ }^{58}$ Although iNOS is beneficial as a host defense mechanism against liver fluke infection, the excessive iNOS formation could lead to oxidative stress and DNA damage, which play an important role in cholangiocarcinogenesis. $^{59}$

In a macrophage cell line (RAW 264.7) study, a crude $O$. viverrini antigen induced the expression of TLR2, NF- $\kappa \mathrm{B}$, iNOS and COX-2 in a dose-dependent manner. ${ }^{60}$ In vivo study showed that $O$. viverrini antigens were found initially in the biliary epitheliums of the intrahepatic and extrahepatic bile ducts. Eventually, the antigens outspreaded into biliary epitheliums of small bile ducts, hepatocytes, Kupffer cells, macrophages, and cells within the egg granuloma. The antigens generated intense inflammatory cells infiltration, particularly those with mononuclear cells. ${ }^{61}$

Regarding $C$. sinensis infection, a mouse model study showed that $C$. sinensis infection upregulated the transcription of TLR2 and TLR4 in endothelial cells, fibroblasts, and biliary epithelium cells resulting in an increase of levels of IL-4, IL-10, tumor necrosis factor $\alpha$ (TNF- $\alpha$ ) and interferon $\gamma \cdot{ }^{62}$ In the affected tissues, there was intense immunoreactivity of lipid peroxidation products. The time-dependently histopathological changes consisted of bile duct epithelial hyperplasia, periductal fibrosis, edema and inflammatory infiltration in infected mice livers. The serum proinflammatory cytokines such as TNF- $\alpha$, IL $\beta-1$ and IL- 6 were also upregulated. ${ }^{63}$

In a human hepatic stellate cell line (LX-2) study, C. sinensis ferritin heavy chain, a component of $C$. sinensis excretory/secretory products (CsESP), increased the production of free radicals resulting in activation of NF- $\kappa \mathrm{B}$ signaling pathway. Correspondingly, the expressions of pro-inflammatory cytokines such as IL $\beta-1$ and IL- 6 were upregulated through NF- $\kappa$ B activation. ${ }^{64,65}$ In a human CCA cell line (HuCCT1) study, CsESP induced the production of intracellular free radicals through upregulation of TLR signaling transduction. ${ }^{66}$ In summary, these immunopathologic mechanisms contribute to CCA development by generating an intense inflammation, advanced periductal fibrosis, and DNA damage.

\section{Parasites' excretory/secretory products-induced cells proliferation}

The parasite excretory/secretory products not only triggered immune-mediated inflammation, but also played many other roles in the carcinogenesis of CCA (Figs 2 and 3). ${ }^{67}$ In a murine fibroblast cell line (NIH-3T3) studies, OvESP promoted cells proliferation $^{68}$ and upregulated genes expression in several pathways, particularly those involving in epidermal growth factor and transforming growth factor $\beta$ pathways. ${ }^{69}$ Data from a proteomic study investigating the entire proteins in OvESP demonstrated that OvESP was a complex blend of proteins associated with cancers, such as granulin, thioredoxin and cystatin. Moreover, many identified surface proteins had no homologues in the public databases yet. ${ }^{70}$ A recent proteomic study of immediate intracellular changes of normal immortalized human cholangiocyte cell line (H69) and human colon cancer cell line (CaCo-2) after incubated with OvESP had shown the molecular mechanisms by which OvESP products interacted with host cells. Kyoto Encyclopedia of Genes and Genomes pathways 


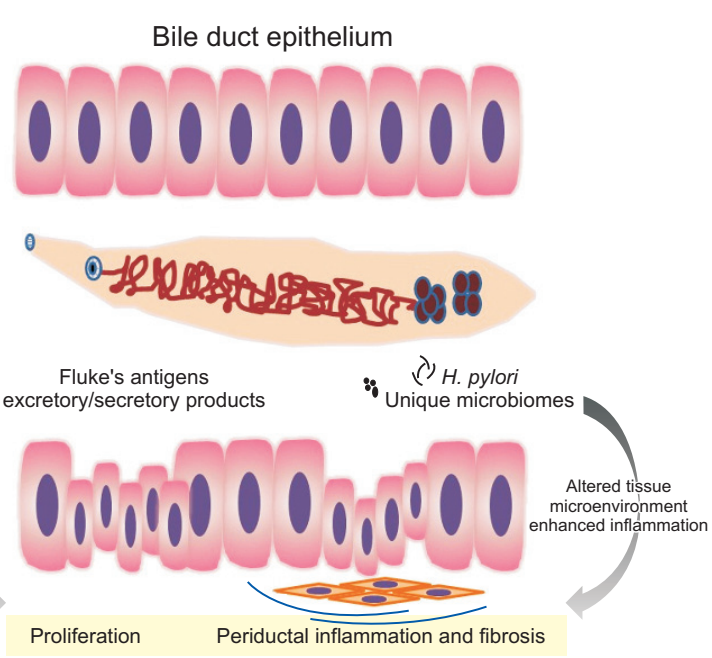

Fig. 3. Cellular reactions to liver fluke antigens, excretory/secretory products and changes in the biliary tract microbiome.

H. pylori, Helicobacter pylori.

analysis identified glycolysis/gluconeogenesis and protein processing in the endoplasmic reticulum as two major differentially induced pathways in $\mathrm{H} 69$ cells compared to CaCo-2 cells. Similarly, in the Reactome pathways analysis, the processes related to the apoptotic execution phase and apoptosis were enriched in H69 cells after incubation with OvESP. ${ }^{47}$

Among various components of OvESP, 0 . viverrini glutathione S-transferase (OvGST) was identified. OvGST had a dose-dependent proliferative effect on murine fibroblasts (NIH-3T3) and non-tumorigenic human bile duct epithelial cells (MMNK1). ${ }^{71}$

O. viverrini granulin (Ov-GRN-1), a homologue of human granulin, was one of the major growth factors in OvESP. OvGRN-1 promoted wound healing at and around the feeding site, which attenuated the host bile duct injury caused by parasites. However, the cells proliferation effect of $0 \mathrm{v}-\mathrm{GRN}-1$ also increased the risk of CCA. In vitro study reported that Ov-GRN-1 stimulated proliferation of murine fibroblasts (NIH-3T3) and a human CCA cell line (KKU-100). ${ }^{72,73}$ Additionally, suppression of Ov-GRN-1 expression by RNA interference reduced the survival of the fluke and the capacity of OvESP to induce proliferation of both human cholangiocyte cell line (H69) and human CCA cell line (KKU-M214). These findings emphasized the importance of Ov-GRN-1 in liver fluke survival and its role in carcinogenesis. ${ }^{74}$

$O$. viverrini thioredoxin $(\mathrm{Ov}-\mathrm{Tr} \mathrm{r}-1)$ was detected in OvESP and in the infected biliary epitheliums. 0v-Trx-1 is an inflammation-inducible oxidoreductase enzyme acting as one of the flukes' defense mechanisms against an oxidative damage caused by a human immune response. ${ }^{75}$ Moreover, $0 v-T r x-1$ could promote cells and tissue growth. In an immortalized human cholangiocyte cell line (H69) study, Ov-Trx-1 inhibited oxidative stress-induced apoptosis of bile duct epithelial cells, which may play a role in carcinogenesis of CCA. ${ }^{76}$

A study of $C$. sinensis showed that CsESP upregulated a num- ber of genes involving in carcinogenesis; and downregulated several apoptosis-inducing genes in the human CCA cell line (HuCCT1). ${ }^{77}$ A study in HuCCT1 cell line found that CsESP increased proliferation of CCA cells and induced the expression of COX-2. Cells pretreated with CsESP were resistant to parthenolide, an anti-inflammatory and anti-cancer agent that induces apoptosis of CCA cells. ${ }^{78}$ An in vitro study of human embryonic kidney epithelial cell line (HEK293) showed that CsESP stimulated cell proliferation by inducing E2F transcription factor 1 (E2F1) expression. ${ }^{79}$ The CsESP upregulated a number of microRNAs regulating cell proliferation and downregulated tumor suppressor microRNAs in both human CCA cell line (HuCCT1) and normal cholangiocyte cell line (H69) which may play a role in carcinogenesis. ${ }^{80}$

\section{Changes in biliary tract microbiome}

In vivo data demonstrated the significant differences in the microbiota within biliary system and feces of $O$. viverrini-infected Syrian hamsters as compared to those of uninfected controls. ${ }^{81}$ The unique microbiome profiles in the bile duct of the infected host influenced tissue microenvironment and contributed to cancer (Fig. 3). ${ }^{82}$ Data from a metagenomic study showed that chronic liver fluke infection augmented bacterial diversity in the liver. Helicobacter pylori were also identified in the liver of the chronic $O$. viverrini-infected hamsters but not in those of uninfected controls. ${ }^{82} O$. viverrini was believed to be a residence of Helicobacter species, and both $O$. viverrini and $H$. pylori may potentially form an obligatory mutualism alliance. ${ }^{83} \mathrm{H}$. pylori, a group 1 carcinogen by International Agency for Research on Cancer (IARC), could contribute to $O$. viverrini-associated CCA by intensifying the degree of inflammation and proliferation of the biliary epitheliums. ${ }^{84,85}$ A hamster model study showed that co-infection of $O$. viverrini and $H$. pylori increased the degree of pathological abnormality, including periductal fibrosis, cholangitis and bile duct hyperplasia, and the mRNA expression levels of IL-1, IL-6 and TNF- $\alpha{ }^{86}$

\section{Posttreatment effect}

A treatment with praziquantel reduced iNOS-dependent DNA damage in $O$. viverrini-infected hamsters by decreased expression of NF- $\kappa \mathrm{B}$ and iNOS in the bile duct epitheliums. ${ }^{87}$ However, the treatment caused a short-term adverse effect due to a sudden release of $O$. viverrini antigens exposing to host immune system resulting in a surge of oxidative and nitrative stress (Fig. 4). ${ }^{88}$ In endemic area, re-infection with liver fluke frequently follow a successful treatment. Thus, a repeating circuit of re-infection and re-treatment could possibly be harmful and can be a part of cholangiocarcinogenesis. ${ }^{89}$ However, this hypothesis was not confirmed by a recent study in hamster model with three cycles of 0 . viverrini infection and treatment with praziquantel. ${ }^{90}$ In summary, the role of repeated treatment on carcinogenesis of CCA remains controversial. 


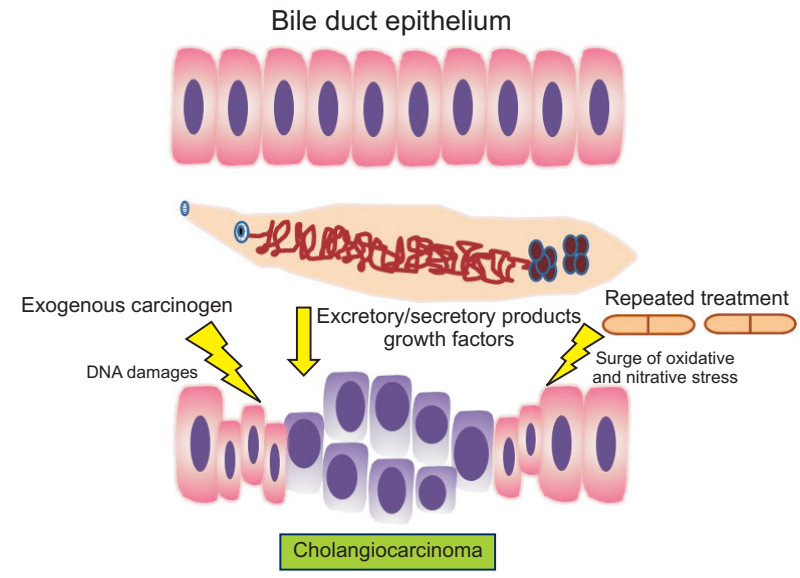

Fig. 4. The cooperation of liver fluke infection, liver fluke treatment, and exogenous carcinogens in the carcinogenesis of cholangiocarcinoma.

\section{FUTURE TREND}

\section{Novel biomarkers}

Recent miRNAomic studies demonstrated the characteristic patterns of miRNA profiles in tissue and in matched plasma in different subtypes of $O$. viverrini-induced intrahepatic CCA. ${ }^{49,50}$ These data pave the way for researchers to better understand mechanisms by which $O$. viverrini initiates cholangiocarcinogenesis. Moreover, the miRNAs are potentially valuable biomarkers that could help as a predictive, diagnostic and prognostic factors of $O$. viverrini infection and $O$. viverrini-related CCA. $^{50}$

\section{Vaccine}

In addition to a miRNAomic data, a genomic and transcriptomic study of $O$. viverrini had illustrated the survival mechanisms of the flukes within the bile duct and how they modulate host cell responses. ${ }^{48}$ Additional fundamental data are needed to unfold the liver fluke vaccine development. Vaccination against liver fluke for people living in endemic areas could potentially reduce the risk of liver fluke-related CCA. ${ }^{10}$

\section{Climate change}

Climate change causes an increasing in our planet temperature continuously. The increasing temperature has a huge effect on vector-borne parasitic diseases. The habitat of the fluke could move northward and southward where are getting warmer while the current habitat around the equator line would be too hot to flourish. ${ }^{91}$ Because of a close association between opisthorchiasis and leptospirosis, the awareness of both diseases should be raised in the potentially migrated habitat area. ${ }^{92}$ The level of precipitation, the change in flowing river and the manmade water reservoirs could also affect the habitat of fishes and snails, which could inevitably affect the prevalence of liver fluke. ${ }^{93,94}$

\section{Public health policy to control liver flukes and impact on prevention of CCA}

Habit of eating undercooked fish and poor sanitation among people in endemic area facilitate the uninterrupted cycle of 0 . viverrini infection. In spite of available treatment with praziquantel, the prevalence of $O$. viverrini is still high as re-infection occurs frequently after treatment. A combination of intensive health education, sanitation improvement, mass stool examination and anthelmintic therapy could be the best way to combat with liver fluke infection which would also result in a decrease of the prevalence of CCA. ${ }^{7,10,95}$ The health education should be both school-based and community-based and is consisting mainly of personal hygiene, especially defecation, and safe cooking. The key to a successful and sustainable control of liver flukes is to have both community along with multi-stakeholder participations. $^{96,97}$

Not only a liver fluke preventive strategy is necessary but a CCA surveillance program should also be performed by ultrasonography, ${ }^{84,85}$ which has a potential to detect, hopefully early, CCA in endemic areas. To date, the benefit of this strategy is still doubtful since is impractical to convince those asymptomatic patients with periductal fibrosis to undergo for liver resection. Moreover, If the detected CCA is at the stage that not amenable for R0 resection, this strategy would cause a lead-time bias. Then, the more reliable novel biomarkers to detect early CCA are the next hope as the currently available markers provide unsatisfied sensitivity and specificity.

\section{CONCLUSIONS}

CCA is an aggressive cancer arising from epithelial cells of the bile duct. Most patients with CCA had unresectable tumor at the time of diagnosis. In Western countries, the risk of CCA increases in patients with primary sclerosing cholangitis whereas liver fluke infection appears to be the major risk factor of CCA in Asian countries. A diagnosis of liver fluke infection often relies on use of stool sample, including microscopic examination, PCR-based assay, and fluke's antigen detection. Tests of serum, saliva and urine sample are also potentially diagnostic. The presence of liver fluke, in concert with exogenous carcinogens, magnifies risk of CCA in people living in endemic areas. The "liver fluke-cholangiocarcinoma" carcinogenesis pathways are consisting of mechanical damages to bile duct epithelium, immunopathologic and cellular reaction to liver fluke's antigens and excretory/secretory products, liver fluke-induced changes in biliary tract microbiome and effect of repeated treatment of liver fluke. Vaccine and novel biomarker are needed for primary and secondary prevention of CCA in endemic area of liver fluke. Finally yet importantly, climate change does have effect on vector-borne parasitic diseases. The awareness of liver fluke should also be raised in the potentially migrated habitat area. 


\section{CONFLICTS OF INTEREST}

No potential conflict of interest relevant to this article was reported.

\section{ACKNOWLEDGEMENTS}

Grant for International Research Integration: Chula Research Scholar, Ratchadaphiseksomphot Endowment Fund.

\section{REFERENCES}

1. Ustundag Y, Bayraktar Y. Cholangiocarcinoma: a compact review of the literature. World J Gastroenterol 2008;14:6458-6466.

2. Jepsen P, Vilstrup H, Tarone RE, Friis S, Sørensen HT. Incidence rates of intra- and extrahepatic cholangiocarcinomas in Denmark from 1978 through 2002. J Natl Cancer Inst 2007;99:895-897.

3. Doherty B, Nambudiri VE, Palmer WC. Update on the diagnosis and treatment of cholangiocarcinoma. Curr Gastroenterol Rep 2017;19:2.

4. Welzel TM, McGlynn KA, Hsing AW, O’Brien TR, Pfeiffer RM. Impact of classification of hilar cholangiocarcinomas (Klatskin tumors) on the incidence of intra- and extrahepatic cholangiocarcinoma in the United States. J Natl Cancer Inst 2006;98:873-875.

5. Gatto M, Bragazzi MC, Semeraro R, et al. Cholangiocarcinoma: update and future perspectives. Dig Liver Dis 2010;42:253-260.

6. Bismuth H, Nakache R, Diamond T. Management strategies in resection for hilar cholangiocarcinoma. Ann Surg 1992;215:31-38.

7. Sripa B, Pairojkul C. Cholangiocarcinoma: lessons from Thailand. Curr Opin Gastroenterol 2008;24:349-356.

8. Ehlken H, Zenouzi R, Schramm C. Risk of cholangiocarcinoma in patients with primary sclerosing cholangitis: diagnosis and surveillance. Curr Opin Gastroenterol 2017;33:78-84.

9. Tyson GL, El-Serag HB. Risk factors for cholangiocarcinoma. Hepatology 2011;54:173-184.

10. Sripa B, Bethony JM, Sithithaworn P, et al. Opisthorchiasis and Opisthorchis-associated cholangiocarcinoma in Thailand and Laos. Acta Trop 2011;120 Suppl 1:S158-S168.

11. Schistosomes, liver flukes and Helicobacter pylori. IARC Working Group on the Evaluation of Carcinogenic Risks to Humans. Lyon, 7-14 June 1994. IARC Monogr Eval Carcinog Risks Hum 1994;61:1-241.

12. Keiser J, Utzinger J. Food-borne trematodiases. Clin Microbiol Rev 2009;22:466-483.

13. Petney T, Sithithaworn P, Andrews R, et al. The ecology of the Bithynia first intermediate hosts of Opisthorchis viverrini. Parasitol Int 2012;61:38-45.

14. Vichasri S, Viyanant V, Upatham ES. Opisthorchis viverrini: intensity and rates of infection in cyprinoid fish from an endemic focus in Northeast Thailand. Southeast Asian J Trop Med Public Health 1982;13:138-141.

15. Yossepowitch 0, Gotesman T, Assous M, Marva E, Zimlichman R,
Dan M. Opisthorchiasis from imported raw fish. Emerg Infect Dis 2004;10:2122-2126.

16. Kaewpitoon N, Kaewpitoon SJ, Pengsaa P, Sripa B. Opisthorchis viverrini: the carcinogenic human liver fluke. World J Gastroenterol 2008;14:666-674.

17. Enes JE, Wages AJ, Malone JB, Tesana S. Prevalence of Opisthorchis viverrini infection in the canine and feline hosts in three villages, Khon Kaen Province, northeastern Thailand. Southeast Asian J Trop Med Public Health 2010;41:36-42.

18. Kaewpitoon N, Kootanavanichpong N, Kompor P, et al. Review and current status of Opisthorchis viverrini infection at the community level in Thailand. Asian Pac J Cancer Prev 2015;16:68256830.

19. Sithithaworn P, Andrews RH, Nguyen VD, et al. The current status of opisthorchiasis and clonorchiasis in the Mekong Basin. Parasitol Int 2012;61:10-16.

20. Sithithaworn P, Haswell-Elkins M. Epidemiology of Opisthorchis viverrini. Acta Trop 2003;88:187-194.

21. Sithithaworn P, Tesana S, Pipitgool V, et al. Relationship between faecal egg count and worm burden of Opisthorchis viverrini in human autopsy cases. Parasitology 1991;102 Pt 2:277-281.

22. Kaewkes S, Elkins DB, Sithithaworn P, Haswell-Elkins MR. Comparative studies on the morphology of the eggs of Opisthorchis viverrini and lecithodendriid trematodes. Southeast Asian J Trop Med Public Health 1991;22:623-630.

23. Chai JY, Han ET, Guk SM, et al. High prevalence of liver and intestinal fluke infections among residents of Savannakhet Province in Laos. Korean J Parasitol 2007;45:213-218.

24. Lee JJ, Jung BK, Lim H, et al. Comparative morphology of minute intestinal fluke eggs that can occur in human stools in the Republic of Korea. Korean J Parasitol 2012;50:207-213.

25. Duenngai K, Sithithaworn P, Rudrappa UK, et al. Improvement of PCR for detection of Opisthorchis viverrini DNA in human stool samples. J Clin Microbiol 2008;46:366-368.

26. Stensvold CR, Saijuntha W, Sithithaworn P, et al. Evaluation of PCR based coprodiagnosis of human opisthorchiasis. Acta Trop 2006;97:26-30.

27. Umesha KR, Kumar S, Parvathi A, et al. Opisthorchis viverrini: detection by polymerase chain reaction (PCR) in human stool samples. Exp Parasitol 2008;120:353-356.

28. Tantrawatpan C, Intapan PM, Thanchomnang T, et al. Development of a PCR assay and pyrosequencing for identification of important human fish-borne trematodes and its potential use for detection in fecal specimens. Parasit Vectors 2014;7:88.

29. Lamaningao P, Kanda S, Laimanivong S, et al. Development of a PCR assay for diagnosing trematode (Opisthorchis and Haplorchis) infections in human stools. Am J Trop Med Hyg 2017;96:221-228.

30. Won EJ, Kim SH, Kee SJ, et al. Multiplex real-time PCR assay targeting eight parasites customized to the Korean population: potential use for detection in diarrheal stool samples from gastroenteritis patients. PLoS One 2016;11:e0166957.

31. Lovis L, Mak TK, Phongluxa K, et al. PCR diagnosis of Opisthorchis 
viverrini and Haplorchis taichui infections in a Lao Community in an area of endemicity and comparison of diagnostic methods for parasitological field surveys. J Clin Microbiol 2009;47:1517-1523.

32. Wongratanacheewin S, Pumidonming W, Sermswan RW, Pipitgool V, Maleewong W. Detection of Opisthorchis viverrini in human stool specimens by PCR. J Clin Microbiol 2002;40:38793880 .

33. Teimoori S, Arimatsu Y, Laha T, et al. Immunodiagnosis of opisthorchiasis using parasite cathepsin F. Parasitol Res 2015;114:45714578.

34. Sirisinha S, Chawengkirttikul R, Haswell-Elkins MR, Elkins DB, Kaewkes S, Sithithaworn P. Evaluation of a monoclonal antibodybased enzyme linked immunosorbent assay for the diagnosis of Opisthorchis viverrini infection in an endemic area. Am J Trop Med Hyg 1995;52:521-524.

35. Watwiengkam N, Sithithaworn J, Duenngai K, et al. Improved performance and quantitative detection of copro-antigens by a monoclonal antibody based ELISA to diagnose human opisthorchiasis. Acta Trop 2013;128:659-665.

36. Nie G, Wang T, Lu S, Liu W, Li Y, Lei J. Detection of Clonorchis sinensis circulating antigen in sera from Chinese patients by immunomagnetic bead ELISA based on IgY. PLoS One 2014;9:e113208.

37. Worasith C, Kamamia C, Yakovleva A, et al. Advances in the diagnosis of human opisthorchiasis: development of Opisthorchis viverrini antigen detection in urine. PLoS Negl Trop Dis 2015;9:e0004157.

38. Sawangsoda P, Sithithaworn J, Tesana S, et al. Diagnostic values of parasite-specific antibody detections in saliva and urine in comparison with serum in opisthorchiasis. Parasitol Int 2012;61:196202.

39. Chaiyarit P, Sithithaworn P, Thuwajit C, Yongvanit P. Detection of salivary antibodies to crude antigens of Opisthorchis viverrini in opisthorchiasis and cholangiocarcinoma patients. Clin Oral Investig 2011;15:477-483.

40. Keiser J, Utzinger J. The drugs we have and the drugs we need against major helminth infections. Adv Parasitol 2010;73:197230.

41. Soukhathammavong P, Odermatt P, Sayasone S, et al. Efficacy and safety of mefloquine, artesunate, mefloquine-artesunate, tribendimidine, and praziquantel in patients with Opisthorchis viverrini: a randomised, exploratory, open-label, phase 2 trial. Lancet Infect Dis 2011;11:110-118.

42. Xu LL, Jiang B, Duan JH, et al. Efficacy and safety of praziquantel, tribendimidine and mebendazole in patients with co-infection of Clonorchis sinensis and other helminths. PLoS Negl Trop Dis 2014;8:e3046.

43. Sayasone $S$, Odermatt $P$, Vonghachack $Y$, et al. Efficacy and safety of tribendimidine against Opisthorchis viverrini: two randomised, parallel-group, single-blind, dose-ranging, phase 2 trials. Lancet Infect Dis 2016;16:1145-1153.

44. Qian MB, Yap P, Yang YC, et al. Efficacy and safety of tribendimidine against Clonorchis sinensis. Clin Infect Dis 2013;56:e76-e82.
45. Jusakul A, Kongpetch S, Teh BT. Genetics of Opisthorchis viverrini-related cholangiocarcinoma. Curr Opin Gastroenterol 2015;31:258-263.

46. Sripa B, Brindley PJ, Mulvenna J, et al. The tumorigenic liver fluke Opisthorchis viverrini: multiple pathways to cancer. Trends Parasitol 2012;28:395-407.

47. Chaiyadet S, Smout M, Laha T, Sripa B, Loukas A, Sotillo J. Proteomic characterization of the internalization of Opisthorchis viverrini excretory/secretory products in human cells. Parasitol Int 2017;66:494-502.

48. Young ND, Nagarajan N, Lin SJ, et al. The Opisthorchis viverrini genome provides insights into life in the bile duct. Nat Commun 2014;5:4378.

49. Peng J, Feng Y, Rinaldi G, et al. The miRNAome of Opisthorchis viverrini induced intrahepatic cholangiocarcinoma. Genom Data 2014;2:274-279.

50. Plieskatt J, Rinaldi G, Feng Y, et al. A microRNA profile associated with Opisthorchis viverrini-induced cholangiocarcinoma in tissue and plasma. BMC Cancer 2015;15:309.

51. Sripa B. Pathobiology of opisthorchiasis: an update. Acta Trop 2003;88:209-220.

52. Zheng S, Zhu Y, Zhao Z, Wu Z, Okanurak K, Lv Z. Liver fluke infection and cholangiocarcinoma: a review. Parasitol Res 2017;116:11-19.

53. Haswell-Elkins MR, Sithithaworn P, Mairiang E, et al. Immune responsiveness and parasite-specific antibody levels in human hepatobiliary disease associated with Opisthorchis viverrini infection. Clin Exp Immunol 1991;84:213-218.

54. Sripa B, Thinkhamrop B, Mairiang E, et al. Elevated plasma IL-6 associates with increased risk of advanced fibrosis and cholangiocarcinoma in individuals infected by Opisthorchis viverrini. PLoS Negl Trop Dis 2012;6:e1654.

55. Sripa B, Mairiang E, Thinkhamrop B, et al. Advanced periductal fibrosis from infection with the carcinogenic human liver fluke Opisthorchis viverrini correlates with elevated levels of interleukin-6. Hepatology 2009;50:1273-1281.

56. Frampton G, Invernizzi P, Bernuzzi F, et al. Interleukin-6-driven progranulin expression increases cholangiocarcinoma growth by an Akt-dependent mechanism. Gut 2012;61:268-277.

57. Chaiyadet S, Smout M, Johnson M, et al. Excretory/secretory products of the carcinogenic liver fluke are endocytosed by human cholangiocytes and drive cell proliferation and IL6 production. Int J Parasitol 2015;45:773-781.

58. Ninlawan K, O'Hara SP, Splinter PL, et al. Opisthorchis viverrini excretory/secretory products induce Toll-like receptor 4 upregulation and production of interleukin 6 and 8 in cholangiocyte. Parasitol Int 2010;59:616-621.

59. Pinlaor S, Hiraku Y, Ma N, et al. Mechanism of NO-mediated oxidative and nitrative DNA damage in hamsters infected with Opisthorchis viverrini: a model of inflammation-mediated carcinogenesis. Nitric Oxide 2004;11:175-183.

60. Pinlaor S, Tada-Oikawa S, Hiraku Y, et al. Opisthorchis viverrini 
antigen induces the expression of Toll-like receptor 2 in macrophage RAW cell line. Int J Parasitol 2005;35:591-596.

61. Sripa B, Kaewkes S. Localisation of parasite antigens and inflammatory responses in experimental opisthorchiasis. Int $\mathrm{J}$ Parasitol 2000;30:735-740.

62. Yan C, Li XY, Li B, et al. Expression of Toll-like receptor (TLR) 2 and TLR4 in the livers of mice infected by Clonorchis sinensis. J Infect Dev Ctries 2015;9:1147-1155.

63. Maeng S, Lee HW, Bashir Q, et al. Oxidative stress-mediated mouse liver lesions caused by Clonorchis sinensis infection. Int $\mathrm{J}$ Parasitol 2016;46:195-204.

64. Mao Q, Xie Z, Wang X, et al. Clonorchis sinensis ferritin heavy chain triggers free radicals and mediates inflammation signaling in human hepatic stellate cells. Parasitol Res 2015;114:659-670.

65. Nam JH, Moon JH, Kim IK, et al. Free radicals enzymatically triggered by Clonorchis sinensis excretory-secretory products cause NF-kappaB-mediated inflammation in human cholangiocarcinoma cells. Int J Parasitol 2012;42:103-113.

66. Bahk YY, Pak JH. Toll-like receptor-mediated free radical generation in Clonorchis sinensis excretory-secretory product-treated cholangiocarcinoma cells. Korean J Parasitol 2016;54:679-684.

67. Chaiyadet S, Sotillo J, Smout M, et al. Carcinogenic liver fluke secretes extracellular vesicles that promote cholangiocytes to adopt a tumorigenic phenotype. J Infect Dis 2015;212:1636-1645.

68. Thuwajit C, Thuwajit P, Kaewkes S, et al. Increased cell proliferation of mouse fibroblast NIH-3T3 in vitro induced by excretory/ secretory product(s) from Opisthorchis viverrini. Parasitology 2004;129(Pt 4):455-464.

69. Thuwajit C, Thuwajit P, Uchida K, et al. Gene expression profiling defined pathways correlated with fibroblast cell proliferation induced by Opisthorchis viverrini excretory/secretory product. World J Gastroenterol 2006;12:3585-3592.

70. Mulvenna J, Sripa B, Brindley PJ, et al. The secreted and surface proteomes of the adult stage of the carcinogenic human liver fluke Opisthorchis viverrini. Proteomics 2010;10:1063-1078.

71. Daorueang D, Thuwajit P, Roitrakul S, et al. Secreted Opisthorchis viverrini glutathione S-transferase regulates cell proliferation through AKT and ERK pathways in cholangiocarcinoma. Parasitol Int 2012;61:155-161.

72. Smout MJ, Laha T, Mulvenna J, et al. A granulin-like growth factor secreted by the carcinogenic liver fluke, Opisthorchis viverrini, promotes proliferation of host cells. PLoS Pathog 2009;5:e1000611.

73. Smout MJ, Mulvenna JP, Jones MK, Loukas A. Expression, refolding and purification of Ov-GRN-1, a granulin-like growth factor from the carcinogenic liver fluke, that causes proliferation of mammalian host cells. Protein Expr Purif 2011;79:263-270.

74. Papatpremsiri A, Smout MJ, Loukas A, Brindley PJ, Sripa B, Laha T. Suppression of Ov-grn-1 encoding granulin of Opisthorchis viverrini inhibits proliferation of biliary epithelial cells. Exp Parasitol 2015;148:17-23.

75. Suttiprapa S, Matchimakul P, Loukas A, et al. Molecular expression and enzymatic characterization of thioredoxin from the carcinogenic human liver fluke Opisthorchis viverrini. Parasitol Int 2012;61:101-106.

76. Matchimakul P, Rinaldi G, Suttiprapa S, et al. Apoptosis of cholangiocytes modulated by thioredoxin of carcinogenic liver fluke. Int J Biochem Cell Biol 2015;65:72-80.

77. Pak JH, Kim DW, Moon JH, et al. Differential gene expression profiling in human cholangiocarcinoma cells treated with Clonorchis sinensis excretory-secretory products. Parasitol Res 2009;104:1035-1046.

78. Kim YJ, Choi MH, Hong ST, Bae YM. Resistance of cholangiocarcinoma cells to parthenolide-induced apoptosis by the excretory-secretory products of Clonorchis sinensis. Parasitol Res 2009;104:1011-1016

79. Kim YJ, Choi MH, Hong ST, Bae YM. Proliferative effects of excretory/secretory products from Clonorchis sinensis on the human epithelial cell line HEK293 via regulation of the transcription factor E2F1. Parasitol Res 2008;102:411-417.

80. Pak JH, Kim IK, Kim SM, et al. Induction of cancer-related microRNA expression profiling using excretory-secretory products of Clonorchis sinensis. Parasitol Res 2014;113:4447-4455.

81. Plieskatt JL, Deenonpoe R, Mulvenna JP, et al. Infection with the carcinogenic liver fluke Opisthorchis viverrini modifies intestinal and biliary microbiome. FASEB J 2013;27:4572-4584.

82. Chng KR, Chan SH, Ng AH, et al. Tissue Microbiome profiling identifies an enrichment of specific enteric bacteria in Opisthorchis viverrini associated cholangiocarcinoma. EBioMedicine 2016;8:195-202.

83. Deenonpoe R, Chomvarin C, Pairojkul C, et al The carcinogenic liver fluke Opisthorchis viverrini is a reservoir for species of Helicobacter. Asian Pac J Cancer Prev 2015;16:1751-1758.

84. Boonyanugomol W, Chomvarin C, Sripa B, et al. Helicobacter pylori in Thai patients with cholangiocarcinoma and its association with biliary inflammation and proliferation. HPB (Oxford) 2012;14:177-184.

85. Sripa B, Deenonpoe R, Brindley PJ. Co-infections with liver fluke and Helicobacter species: a paradigm change in pathogenesis of opisthorchiasis and cholangiocarcinoma? Parasitol Int 2017;66:383-389.

86. Dangtakot R, Pinlaor S, Itthitaetrakool U, et al. Coinfection with Helicobacter pylori and Opisthorchis viverrini enhances the severity of hepatobiliary abnormalities in hamsters. Infect Immun 2017;85:e00009-e00017.

87. Pinlaor S, Hiraku Y, Yongvanit P, et al. iNOS-dependent DNA damage via NF-kappaB expression in hamsters infected with Opisthorchis viverrini and its suppression by the antihelminthic drug praziquantel. Int J Cancer 2006;119:1067-1072.

88. Pinlaor S, Prakobwong S, Hiraku Y, et al. Oxidative and nitrative stress in Opisthorchis viverrini-infected hamsters: an indirect effect after praziquantel treatment. Am J Trop Med Hyg 2008;78:564573.

89. Kamsa-Ard S, Luvira V, Pugkhem A, et al. Association between praziquantel treatment and cholangiocarcinoma: a hospital-based 
matched case-control study. BMC Cancer 2015;15:776.

90. Hanpanich P, Laha T, Sripa B, et al. Decreased risk of cholangiocarcinogenesis following repeated cycles of Opisthorchis viverrini infection-praziquantel treatment: magnetic resonance imaging (MRI) and histopathological study in a hamster model. Parasitol Int 2017;66:464-470.

91. Suwannatrai A, Pratumchart K, Suwannatrai K, et al. Modeling impacts of climate change on the potential distribution of the carcinogenic liver fluke, Opisthorchis viverrini, in Thailand. Parasitol Res 2017;116:243-250.

92. Van CD, Doungchawee G, Suttiprapa S, Arimatsu Y, Kaewkes S, Sripa B. Association between Opisthorchis viverrini and Leptospira spp. infection in endemic Northeast Thailand. Parasitol Int 2017;66:503-509.

93. Ong X, Wang YC, Sithithaworn P, Namsanor J, Taylor D, Laithavewat L. Uncovering the pathogenic landscape of helminth (Opisthorchis viverrini) infections: a cross-sectional study on contributions of physical and social environment and healthcare interventions. PLoS Negl Trop Dis 2016;10:e0005175.

94. Kim CS, Echaubard P, Suwannatrai A, Kaewkes S, Wilcox BA, Sripa B. Seasonal and spatial environmental influence on Opisthorchis viverrini intermediate hosts, abundance, and distribution: insights on transmission dynamics and sustainable control. PLoS Negl Trop Dis 2016;10:e0005121.

95. Kamsa-ard S, Wiangnon S, Suwanrungruang K, et al. Trends in liver cancer incidence between 1985 and 2009, Khon Kaen, Thailand: cholangiocarcinoma. Asian Pac J Cancer Prev 2011;12:22092213.

96. Sripa B, Tangkawattana S, Laha T, et al. Toward integrated opisthorchiasis control in northeast Thailand: the Lawa project. Acta Trop 2015;141(Pt B):361-367.

97. Sripa B, Tangkawattana S, Sangnikul T. The Lawa model: a sustainable, integrated opisthorchiasis control program using the EcoHealth approach in the Lawa Lake region of Thailand. Parasitol Int 2017;66:346-354. 